\title{
Ruling out the Weinberg Model of Spontaneous CP Violation
}

\author{
${ }^{1,4}$ Darwin Chang, ${ }^{2,3}$ Xiao-Gang He, and ${ }^{3}$ Bruce H.J. McKellar \\ ${ }^{1}$ Department of Physics, National Tsinhua University, Hsinchu, 300, Taiwan. \\ 2 Department of Physics, National Taiwan University, Taipei, 10617, Taiwan. \\ ${ }^{3}$ School of Physics, University of Melbourne, Parkville, Vic 3052, Australia. \\ and \\ ${ }^{4}$ SLAC, Stanford, CA 94309, USA.
}

(August 1999, revised August 2000)

\begin{abstract}
There have been many declarations of the death of the Weinberg model of spontaneous CP violation. Previous studies, before the recent measurements of $\epsilon^{\prime} / \epsilon$, indicated that the model could not accommodate the experimental values on $\epsilon$ in $K^{0}-\bar{K}^{0}$ mixing, the neutron electric dipole moment (EDM), the branching ratio of $b \rightarrow s \gamma$ and the upper limit on $\epsilon^{\prime} / \epsilon$. We point out that these studies were based on optimistic estimates of the uncertainties in the calculations and that when more realistic estimates of these errors are used the Weinberg model cannot be conclusively ruled out from these considerations alone.

Here we use these realistic error estimates to analyze the present situation of the Weinberg model. The latest results from Belle and BaBar on $\sin 2 \beta$ allow the small values of this parameter which occur naturally in the Weinberg model. However, in this model, the recently measured value of $\operatorname{Re}\left(\epsilon^{\prime} / \epsilon\right)=$ $(1.92 \pm 0.25) \times 10^{-3}$ cannot be made compatible with the branching ratio
\end{abstract}


$B(b \rightarrow s \gamma)=(3.15 \pm 0.54) \times 10^{-4}$. As a result we conclude that the Weinberg model is now confidently and conservatively ruled out.

11.30.-j, 12.60.Fr, 12.15.-y

Typeset using REVTEX 


\section{INTRODUCTION}

The origin of $\mathrm{CP}$ violation remains one of the outstanding problems of modern particle physics. Although the Standard Model (SM) of CP violation based on the KobayashiMaskawa (KM) mechanism is consistent [1] with observations of $\mathrm{CP}$ violation in $K_{S}$ and $K_{L}$ mixing [2] and in $K_{S, L} \rightarrow \pi \pi$ decay amplitudes [3], there are intriguing hints, from consideration of baryon asymmetry of the universe [4], that other sources of $\mathrm{CP}$ violation may exist. These non-standard sources of CP violation could occur as well as, or instead of, the SM source.

Models based on additional Higgs bosons [5,6] provide alternatives which explain the existing laboratory data [7] and produce the large $\mathrm{CP}$ violation required for baryon asymmetry [4]. Such models also allow CP symmetry to be broken spontaneously [6]:8] and therefore give an interesting explanation of the origin for $\mathrm{CP}$ violation. The minimal model of this type satisfying the requirement of vanishing tree level flavour changing neutral current ("natural flavor conservation") is a model of spontaneous CP violation with three Higgs doublets proposed by Weinberg [6] and refined by Branco [8]. We shall refer to it as Weinberg model from here on.

It has frequently been claimed that the Weinberg model is in conflict with the data on the following:

- the value of $\sin 2 \beta$.

- the ratio $\epsilon^{\prime} / \epsilon$,

- the $\epsilon$ parameter in $K^{0}-\bar{K}^{0}$ mixing,

- the neutron electric dipole moment (EDM),

- the branching ratio of $b \rightarrow s \gamma$,

First of all, the Weinberg model predicts small values (less than 0.05) for the parameter $\sin 2 \beta$ which here we define as the parameter that characterizes the $\mathrm{CP}$ violation in $B \rightarrow$ 
$J / \psi K_{S}$ decays [9,10]. The present results [11] from Belle $\left(0.45_{-0.44}^{+0.43}(\text { stat })_{-0.09}^{+0.07}(\right.$ sys $\left.)\right)$ and BaBar $(0.12 \pm 0.37 \pm 0.09)$ on $\sin 2 \beta$ allow such small values of this parameter, although the earlier combined result from ALEPH, OPAL and $\mathrm{CDF}(0.91 \pm 0.35)$ [12] favoured larger values. Therefore these considerations do not rule out the Weinberg model, and to determine whether or not the model is consistent with present day data we have to turn to the other observables.

In early discussions of $\mathrm{CP}$ violation in the neutral kaon system, it was assumed that only the short-distance contributions from the $\mathrm{CP}$ violating $\Delta S=2$ box diagrams, due to either two charged Higgs particles, or one charged Higgs plus one $W$ exchange, are responsible for the measured value of $\epsilon$, then, since the charged Higgs couplings to light fermions are proportional to the fermion masses a very large $\mathrm{CP}$ violating coupling is required to fit the data. If this same $\mathrm{CP}$ violating parameter is then used for the calculation of $\epsilon^{\prime} / \epsilon$, the contribution is much larger than the experimental value [13, 14]. It was later shown that there are important long distance contributions to $\epsilon$ due to the Higgs induced $\Delta s=1$ $\mathrm{CP}$ violating operators, and that if these are taken into account, the model can be made consistent with the observed $\epsilon$ and $\epsilon^{\prime} / \epsilon$ [15, 16].

Previous studies have claimed that although the Weinberg model is consistent with CP violation in kaon system, it has problems with the neutron electric dipole moment (EDM) [17] and the branching ratio for $b \rightarrow s \gamma$ [9, 10]. These analyses have relied on optimistic estimates of the accuracy of the calculations of relevant hadronic matrix elements [18].

After briefly describing the Weinberg model of $\mathrm{CP}$ violation, we review the current experimental and theoretical status of each of these observables individually, and then discuss the constraints placed on the Weinberg model by the total ensemble of data. We find that it is possible to rule out this model definitively only if one imposes simultaneously the new constraints from the recently measured value of $\operatorname{Re}\left(\epsilon^{\prime} / \epsilon\right)=(1.92 \pm 0.25) \times 10^{-3}$ [3] and the branching ratio $B(b \rightarrow s \gamma)=(3.15 \pm 0.54) \times 10^{-4}$ [19].

Therefore, because of the stronger constraints imposed by recent data, one can now 
declare that the Weinberg model of spontaneous CP violation is ruled out in spite of the relatively large hadronic uncertainty.

\section{THE WEINBERG MODEL OF CP VIOLATION}

In the Weinberg model, three Higgs doublets are introduced. The spontaneous breakdown of gauge symmetry then induces massive Higgs eigenstates for the charged Higgs particles, and introduces a mixing matrix specifying the interaction eigenstates of the Higgs doublets in term of the mass eigenstates. Being a $3 \times 3$ mixing matrix between charged particles, the matrix contains exactly one irreducible complex phase, thus inducing CP violation. After spontaneous symmetry breaking, there are two physical charged and five neutral Higgs particles. As CP violation in flavour changing processes in this model is dominated by exchange of charged Higgs particles, we concentrate our attentioin on this contribution. The interaction Lagrangian for the coupling of the two charged Higgs $\left(H_{1}^{+}\right.$and $\left.H_{2}^{+}\right)$to fermions 20] can be written as

$$
L=2^{3 / 4} G_{F}^{1 / 2} \bar{U}\left[V_{K M} M_{D}\left(\alpha_{1} H_{1}^{+}+\alpha_{2} H^{+}\right) R+M_{U} V_{K M}\left(\beta_{1} H_{1}^{+}+\beta_{2} H_{2}^{+}\right) L\right] D+H . C .
$$

where $R(L)=\left(1 \pm \gamma_{5}\right) / 2$, and $M_{U, D}$ are the diagonal up and down quark mass matrices. The parameters $\alpha_{i}$ and $\beta_{i}$, which satisfy $\operatorname{Im}\left(\alpha_{1} \beta_{1}^{*}\right)=-\operatorname{Im}\left(\alpha_{2} \beta_{2}^{*}\right)$, are obtained from the diagonalisation of the charged Higgs mass matrix . The KM matrix elements $V_{i j}$ can be made all real at tree level as a consequence of spontaneous CP violation.

\section{III. $\sin 2 \beta$ IN THE WEINBERG MODEL}

It is well known that $\mathrm{CP}$ violation in B decays will eventually provide crucial constraints on models of $\mathrm{CP}$ violation when sufficient data is available. This is especially true of the gold-plated mode $B \rightarrow J / \psi K_{S}$.

In the Weinberg model, CP violating contributions to the decay amplitudes and to $B^{0}-\bar{B}^{0}$ are both proportional to $\operatorname{Im}\left(\alpha_{1} \beta_{1}^{*}\right)$. The Higgs contributions to the decay amplitudes 
are suppressed, relative to the SM contributions, by additional factors of $m_{c} m_{b} / m_{H}^{2}$, while the mixing is supressed by a factor of $m_{b}^{2} / m_{H}^{2}$. These suppression factors lead to small CP violating phases and result in a very small value for $\sin 2 \beta$ [9, 10], $|\sin 2 \beta|<0.05$.

The ALEPH, OPAL and CDF data reported in 1999 gave $\sin 2 \beta=0.91 \pm 0.35$ [12] which is in conflict with the above limit at the $2 \sigma$ level. However at ICHEP2000, Belle and BaBar reported preliminary results [11], which when averaged with the above, give $\sin 2 \beta=0.49 \pm 0.45$, consistent with the above limit at the $75 \%$ level. Thus the present $\sin 2 \beta$ measurements do not rule out the Weinberg model.

\section{IV. $\epsilon^{\prime} / \epsilon$ IN THE WEINBERG MODEL}

The dominant contribution to $\epsilon^{\prime} / \epsilon$ in the Weinberg model is from the flavor changing gluonic dipole interaction given by 9

$$
\begin{aligned}
& H(s d g)=i g_{s} \tilde{f} m_{s} \bar{s} \sigma_{\mu \nu} \lambda^{a} G_{a}^{\mu \nu}\left(1-\gamma_{5}\right) d \\
& \tilde{f}=\frac{G_{F}}{\sqrt{2}} \frac{1}{16 \pi^{2}} V_{i s} V_{i d} \operatorname{Im}\left(\alpha_{1}^{*} \beta_{1}\right)\left(F_{3}\left(m_{i}^{2} / m_{H_{1}}^{2}\right)-F_{3}\left(m_{i}^{2} / m_{H_{2}}^{2}\right)\right) \eta_{g}, \\
& F_{3}(x)=\frac{1}{2} \frac{x}{(1-x)^{3}}\left[-\frac{3}{2}+2 x-\frac{1}{2} x^{2}-\ln x\right],
\end{aligned}
$$

where $i$ is summed over $u, c, t$ and $\eta_{g}=\left(\alpha_{s}\left(m_{H}\right) / \alpha_{s}(\mu)\right)^{14 /\left(33-2 n_{f}\right)}$ is the one loop QCD correction factor [21] in which $n_{f}$ being the number of quark with mass less than $\mu$. To obtain this correction factor we will use one loop running for $\alpha_{s}$ with the starting value $\alpha_{s}\left(m_{Z}\right)=0.119$. The contribution to $\epsilon^{\prime} / \epsilon$ is dominated by the lightest charged Higgs exchange. In our later discussions, we will assume $H_{1}^{+}$is the lighter one and the other is very heavy and its effects can be neglected.

Theoretical analyses for $\epsilon^{\prime} / \epsilon$ are conventionally carried out in terms of the isospin amplitude $A_{I}$ for $K \rightarrow \pi \pi$. Expressing $\epsilon^{\prime} / \epsilon$ in terms of $A_{I}$, one obtains

$$
\operatorname{Re}\left(\frac{\epsilon^{\prime}}{\epsilon}\right) \approx \frac{\omega}{\sqrt{2}|\epsilon|}\left(\frac{\operatorname{Im} A_{2}}{\operatorname{Re} A_{2}}-\frac{\operatorname{Im} A_{0}}{\operatorname{Re} A_{0}}\right),
$$

where $\omega=\operatorname{Re} A_{2} / \operatorname{Re} A_{0} \approx 1 / 22.2$. 
The dominant gluon dipole operator Eq (21) generates a non-zero value only for $A_{0}$. Calculating the decay amplitudes is our most difficult task, because of our poor understanding of the strong interaction at low energies. Theoretical calculations for the real part of the amplitudes can be easily off by a factor of two to three. For this reason we use the experimental value for $\operatorname{Re} A_{0}=33.3 \times 10^{-8} \mathrm{GeV}^{-2}$ to minimize the error in the calculation of $\epsilon^{\prime} / \epsilon$. But we still have to calculate $\operatorname{Im} A_{0}$. This requires the evaluation of the matrix element $\left\langle(\pi \pi)_{0}|\mathcal{O}| K\right\rangle$. Here $(\pi \pi)_{0}$ indicates the isospin $I=0$ component and $\mathcal{O}=g_{s} m_{s} \bar{s} \lambda^{a} \sigma_{\mu \nu} G_{a}^{\mu \nu}\left(1-\gamma_{5}\right) d$.

A naive PCAC calculation 22,23] gives

$$
\left\langle(\pi \pi)_{0}|\mathcal{O}| K\right\rangle=-2 \sqrt{3 / 2}\left(m_{0}^{2} m_{s} /\left(m_{u}+m_{s}\right)\right)\left(m_{K}^{2} f_{K} / f_{\pi}\right)
$$

with $m_{0}^{2} \approx 1 \mathrm{GeV}^{2}$. A bag model calculation [14] of

$$
A_{K \pi}=<\pi^{0}\left|\bar{s} \lambda^{a} \sigma_{\mu \nu} G_{a}^{\mu \nu}\left(1-\gamma_{5}\right) d\right| K>
$$

gives

$$
A_{K \pi} \approx 0.4 \mathrm{GeV}^{3}
$$

and with the use of current algebra this [16] gives a value for $\left\langle(\pi \pi)_{0}|\mathcal{O}| K\right\rangle$ similar to that of $\mathrm{Eq}$ (四).

It was later realized that the above result is incorrect because an important "tadpole" contribution due to the K-vacuum transition caused by the same operator had been neglected. This contribution cancels the above PCAC result exactly [16,22]. In a chiral perturbation theory approach, this means that the leading order contribution vanishes as expected from the Feinberg-Kabir-Weinberg Theorem [16]. A non-zero value for $\left\langle(\pi \pi)_{0}|\mathcal{O}| K\right\rangle$ can only be generated at $p^{4}$ order in chiral perturbation theory, and can be estimated to be [23]

$$
<(\pi \pi)_{0}|\mathcal{O}| K>=-11 \sqrt{\frac{3}{2}} \frac{m_{s}}{m_{s}+m_{d}} \frac{f_{K}^{2}}{f_{\pi}^{3}} m_{K}^{2} m_{\pi}^{2} B_{0},
$$

where $B_{0}$ is a fudge factor representing the potential uncertainty in the above estimate. We assume that $B_{0}$ is of order 1 . 
Using this matrix element, we obtain

$$
\begin{aligned}
\operatorname{Re}\left(\frac{\epsilon^{\prime}}{\epsilon}\right) & =\frac{\omega}{\sqrt{2}|\epsilon| \operatorname{Re} A_{0}} 11 \sqrt{\frac{3}{2}} \frac{m_{s}}{m_{s}+m_{d}} \frac{f_{K}^{2}}{f_{\pi}^{3}} m_{K}^{2} m_{\pi}^{2} \tilde{f} B_{0}\left(1-\Omega_{\eta+\eta^{\prime}}\right) \\
& =1.7 \times 10^{7}\left(\mathrm{GeV}^{2}\right) \tilde{f} B_{0}
\end{aligned}
$$

where the numerical value follows from the experimental values for $\epsilon$ and $R e A_{0}$, and the isospin breaking correction factor $\Omega_{\eta+\eta^{\prime}}=0.25$ given in ref [24]

To produce the recently observed value for $\epsilon^{\prime} / \epsilon$ within $3 \sigma, \tilde{f} B_{0}$ has to be in the range $+(0.69 \sim 1.57) \times 10^{-10} \mathrm{GeV}^{-2}$. For a given Higgs mass, the $\mathrm{CP}$ violating parameter $\operatorname{Im}\left(\alpha_{1} \beta_{1}^{*}\right)$

is determined by the value of $\tilde{f}$. Only $\tilde{f} B_{0}$ is determined by the data. Remember that the different leading order contributions cancel each other. However, numerically the value obtained from Eq (7) with $B_{0}=1$ is not much smaller than the individual leading terms before cancellations, suggesting that $B_{0}=1$ is the maximum value of $B_{0}$ and hence that the corresponding low value for $\tilde{f}, \tilde{f}=0.69 \times 10^{-10} \mathrm{GeV}^{-2}$, represents its probable lower bound. Nevertheless, we conservatively allow $B_{0}$ to vary from 0.5 to 2 in our estimate to account for possible uncertainties [25]. The most conservative range for $\tilde{f}$ is then $(0.35 \sim 3.1) \times 10^{-10}$ $\mathrm{GeV}^{-2}$. Thus $\tilde{f}$ smaller than $0.35 \times 10^{-10} \mathrm{GeV}^{-2}$ in magnitude is unlikely to generate $\epsilon^{\prime} / \epsilon$ as large as observed. Note that $\tilde{f}$ is positive because $B_{0}$, calculated in Ref [23, is positive.

\section{V. $\epsilon$ IN THE WEINBERG MODEL}

A successful model for $\mathrm{CP}$ violation must to be able to produce the experimental value for $\epsilon$. In this model the short distance $\Delta S=2$ interaction gives too small a value for $\epsilon$, and the dominant contribution actually comes from long distance effects, which in turn are generated by CP violation due to the gluonic dipole interaction. Following Ref. [16] we assume the contribution to $\epsilon$ is from $\pi, \eta, \eta^{\prime}$ poles with one $\mathrm{CP}$ conserving and one $\mathrm{CP}$ violating $\mathrm{K}$ to $\pi, \eta, \eta^{\prime}$ transition. One has [16]

$$
|\epsilon|=\frac{\tilde{f} \kappa g_{s} m_{s} A_{K \pi} H_{\pi K}}{\sqrt{2} m_{K} \Delta m_{L-S}\left(m_{K}^{2}-m_{\pi}^{2}\right)},
$$




$$
\begin{aligned}
\kappa= & 1+\frac{m_{K}^{2}-m_{\pi}^{2}}{m_{K}^{2}-m_{\eta}^{2}}\left[\sqrt{\frac{1}{3}}(1+\delta) \cos \theta+2 \sqrt{\frac{2}{3}} \rho \sin \theta\right]^{2} \\
& +\frac{m_{K}^{2}-m_{\pi}^{2}}{m_{K}^{2}-m_{\eta^{\prime}}^{2}}\left[\sqrt{\frac{1}{3}}(1+\delta) \sin \theta-2 \sqrt{\frac{2}{3}} \rho \cos \theta\right]^{2},
\end{aligned}
$$

where $\Delta m_{L-S}$ is the mass difference of the long and short lived neutral kaons, $\theta$ is the $\eta-\eta^{\prime}$ mixing angle, and $\delta$ and $\rho$ parameterize $\mathrm{SU}(3)$ and $\mathrm{U}(3)$ breaking effects, respectively. In the $\mathrm{SU}(3)$ limit, $\delta=0$; in the $\mathrm{U}(3)$ limit, $\rho=1$. $H_{\pi K}$ is the $\mathrm{CP}$ conserving $\Delta S=1, K-\pi$ transition amplitude which is determined from current algebra to be 16$] H_{\pi K}=2.578 \times 10^{-8}$ $\mathrm{GeV}^{2} . \tilde{H}_{\pi K}=\tilde{f} g_{s} m_{s} A_{K \pi}$ is the $\mathrm{CP}$ violating $K-\pi$ transition amplitude due to the gluonic dipole interaction, expressed in terms of the matrix element $A_{K \pi}$ of Eq (5). Here the QCD coupling constant $g_{s}$ is evaluated at the Kaon scale and is not well determined. Following Ref. [26], we use $g_{s}=4 \pi / \sqrt{6}$ in the matrix element calculation. For $m_{s}$, one should take the values used in conjunction with the models used to calculate relevant matrix elements, for example in the bag model calculation it is in the range of 0.3 to $0.5 \mathrm{GeV}$ [27].

The parameters, $\theta, \delta, \rho$, and the theoretical calculation of $A_{K \pi}$, introduce uncertainties. At present, there are two possible values $-11^{\circ}$ and $-22^{\circ}$ [28] for the mixing angle $\theta$. The $\mathrm{SU}(3)$ breaking parameter $\delta$ is theoretically estimated [29]. The best fit [30] $K_{L} \rightarrow \gamma \gamma$ and $K \rightarrow \pi \pi \gamma$ gives a $\mathrm{U}(3)$ breaking parameter $\rho=0.78$. However, $\rho$ in the range of $0.7 \sim 1.3$ is not ruled out. Using $\theta=-22^{\circ}, \delta=0.17$ and $\rho=0.78$, one obtains $\kappa=0.2$. Most of the previous calculations used this value for $\kappa$. However, the value for $\kappa$ is very sensitive to the specific values of the parameters involved, for example, with $\theta=-11^{\circ}\left(-22^{\circ}\right), \rho=1.3$, and $\delta=0.17(0.0), \kappa$ is approximately $-0.9(-0.95)$. The magnitude of $\kappa$ can change by a factor of four or five. Within the allowed parameter space, $\kappa$ can vary between 0.2 to -1.0 . We note that the sign of $\kappa$ changes in the allowed range of parameters which implies that the relative sign of $\epsilon$ and $\epsilon^{\prime}$ can change.

The uncertainty in the value $A_{\pi K}$ is also quite large. A bag model calculation gives $A_{\pi K}=$ $0.4 \mathrm{GeV}^{3}$ [14]. Major sources of uncertainty include the determination of the numerical values of $\alpha_{s}$ at the kaon decay scale, of the bag radius $R$ and of the strange quark mass $m_{s}$ in bag 
model [27]. A factor of two to three times increase in $A_{\pi K}$ is not ruled out. In view of these uncertainties, we consider $\left(\kappa, A_{K \pi}\right)$ in the rectangle with corners $\left(0.2,0.4 \mathrm{GeV}^{3}\right)$ and $\left(-1.0,1.2 \mathrm{GeV}^{3}\right)$ to be allowed by present experimental and theoretical estimates. Of the two extreme values are, set a) $\left(0.2,0.4 \mathrm{GeV}^{3}\right)$ and set b) $\left(-1.0,1.2 \mathrm{GeV}^{3}\right)$, set a) is the mostly used one in the literature, while set b) represents the most conservative values for $\kappa$ and $A_{K \pi}$.

We find that if the parameters of set a) are used to fit $\epsilon$, the parameter $\tilde{f}$ is determined to be $2.56 \times 10^{-10} \mathrm{GeV}^{-2}$. However, if set b) is used, $\tilde{f}$ can be negative and as small as $0.17 \times 10^{-10} \mathrm{GeV}^{-2}$ in magnitude. There are solutions for $\epsilon$ with $\tilde{f}$ in the ranges $(0.85 \sim$ $2.56) \times 10^{-10} \mathrm{GeV}^{-2}$ and also with $\tilde{f}$ near $-0.17 \times 10^{-10} \mathrm{GeV}^{-2}$. The allowed range of $\tilde{f}$ associated with $\epsilon$ is thus quite large, and it has a large overlap with that determined from $\epsilon^{\prime} / \epsilon$ for positive $\tilde{f}$.

\section{THE NEUTRON ELECTRIC DIPOLE MOMENT}

The experimental bound on the neutron EDM, $d_{n}$, has been used to provide restrictions on the model, and has been claimed to rule it out [17]. The neutron EDM can be generated by the exchange of neutral and charged Higgs particles [31 34. It is not impossible that these contributions may cancel each other and result in a very small neutron EDM. Here we will not entertain this possibility. We will instead single out the variously potentially large valence quark contributions and require that each of them satisfies the experimental constraints.

The contribution of charged Higgs exchange to the neutron EDM is well constrained by fixing the $\mathrm{CP}$ violating parameter $\operatorname{Im}\left(\alpha_{1} \beta_{1}^{*}\right)$ to fit $\epsilon^{\prime} / \epsilon$ and $\epsilon$. The contributions from neutral Higgs exchange are much less constrained. Even in the charged Higgs case we need to use

the theoretical expression for $\tilde{f}$ to extract $\operatorname{Im}\left(\alpha_{1} \beta_{1}^{*}\right)$, and this introduces a sensitivity to the values of the KM elements because the internal charm and top contributions are comparable and can add constructively or destructively depending on the relative sign of combinations 
of the KM matrix elements. This also introduces uncertainties in the calculations. The case where the contributions tend to cancel will result in a large $\operatorname{Im}\left(\alpha_{1} \beta_{1}^{*}\right)$ and lead to difficulties with other data as discussed below. We will use values of the KM matrix elements within the errors given in Ref. [2] such that terms contribute constructively. Specifically, for later discussions, we use $V_{u d}=0.9741, V_{u s}=0.221, V_{c d}=-0.220, V_{c s}=0.9740$ and $V_{t s}=-0.040$.

The charged Higgs boson contribution to the neutron EDM is strongly restricted. The dominant term comes from the down quark EDM. Using the valence quark model, we have

$$
\begin{aligned}
& d_{n} \approx \frac{4}{3} d_{d}=\frac{8}{3} e m_{d} \tilde{f} \frac{V_{c d}^{2} F_{2}\left(m_{c}^{2} / m_{H_{1}}^{2}\right)+\eta_{\gamma} V_{t d}^{2} F_{2}\left(m_{t}^{2} / m_{H_{1}}^{2}\right)}{V_{c d} V_{c s} F_{3}\left(m_{c}^{2} / m_{H_{1}}^{2}\right)+\eta_{g} V_{t d} V_{t s} F_{3}\left(m_{t}^{2} / m_{H_{1}}^{2}\right)}, \\
& F_{2}(x)=-\frac{x}{6(1-x)^{3}}[(3-5 x)(1-x)+(4-6 x) \ln x] .
\end{aligned}
$$

Here we have neglected the small QCD correction to the electric dipole operator from the gluonic dipole operator induced by operator mixing. The leading QCD correction factor for the electric dipole operator is given by $[21] \eta_{\gamma}=\left[\alpha_{s}\left(m_{W}\right) / \alpha_{s}(\mu)\right]^{16 /\left(33-2 n_{f}\right)}$.

Using $\tilde{f}=(0.35 \sim 3.1) \times 10^{-10} \mathrm{GeV}^{-2}$ determined from $\epsilon^{\prime} / \epsilon$, and alowing the lightest charged Higgs mass to range from its lower bound around $70 \mathrm{GeV}$ to several hundred $\mathrm{GeV}$, we estimate the charged Higgs contribution to the neutron EDM as $(0.25 \sim$ $3.5) \times 10^{-24}\left(m_{d} / 300 \mathrm{MeV}\right)$ ecm. Note that $d_{n}$ is proportional to the light quark mass. This introduces a further uncertainty because it is not clear whether the current or the constituent mass should be used. There are also other uncertainties due to the off shell nature of the quarks 35.

The neutral Higgs boson exchange gives a contribution which is not well determined. Even the sign of the contribution is unknown. Since it is not related to the other parameters we have introduced, and since a wide range of the parameter $\tilde{f}$ is still allowed, we will not consider the possible contributions from exchange of neutral Higgs bosons in our estimates. 


\section{VII. $B \rightarrow S \gamma$ IN THE WEINBERG MODEL}

The CP conserving process $b \rightarrow s \gamma$ can place constraints on the CP violating parameters of the model [9, 10], because the CP violating amplitudes contribute to the total rate. In the Weinberg model, although $d s \gamma$ interaction is constrained to be small, the corresponding $b s \gamma$ interaction is enhanced by a factor of $\left(\sim m_{t}^{2} m_{b} / m_{c}^{2} m_{s}\right)\left(V_{t b} V_{t s} / V_{c s} V_{c d}\right) \sim 10^{5}$. Due to this enhancement factor, the predicted branching ratio of $b \rightarrow s \gamma$ may be in conflict with experimental data. Using the leading log result and normalizing the branching ratio due to charged Higgs contribution to the SM one, we have 36]

$$
\begin{aligned}
& \operatorname{Br}(b \rightarrow s \gamma)=7.1 \times 10^{-4}\left[\left(0.313+0.273 r_{1}\right)^{2}+\left(0.273 r_{2}\right)^{2}\right], \\
& r_{1}=1+\frac{\left|\beta_{1}\right|^{2} F_{1}\left(m_{t}^{2} / m_{H_{1}}^{2}\right) / 3-\operatorname{Re}\left(\alpha_{1} \beta_{1}^{*}\right) F_{2}\left(m_{t}^{2} / m_{H_{1}}^{2}\right)}{F_{1}\left(m_{t}^{2} / m_{W}^{2}\right)}, \\
& r_{2}=-\frac{\operatorname{Im}\left(\alpha_{1} \beta_{1}^{*}\right) F_{2}\left(m_{t}^{2} / m_{H_{1}}^{2}\right)}{F_{1}\left(m_{t}^{2} / m_{W}^{2}\right)}, \\
& F_{1}(x)=\frac{x}{12(1-x)^{4}}\left[\left(7-5 x-8 x^{2}\right)(1-x)+x(12-18 x) \ln x\right] .
\end{aligned}
$$

In the above we have neglected the small contribution from the gluonic $b s g$ interaction. CP conserving amplitudes generate the first term in the brackets, and $r_{1}$ contains all of the contributions dependent on $m_{t}$. There are both SM and Weinberg model contributions, and there is a region in parameter space where that the CP conserving contributions of the SM and of charged Higgs exchange mutually cancel. The CP violating amplitudes generate $r_{2}$, which contributes significantly. The branching ratio increases with Higgs mass for fixed $\tilde{f}$.

The experimental branching ratio 19$], B(b \rightarrow s \gamma)=(3.15 \pm 0.54) \times 10^{-4}$, has recently been confirmed by Belle [11]. For the 95\% c.l. upper bound $4.5 \times 10^{-4}$ for $b \rightarrow s \gamma$ [19, we find that there are solutions with $|\tilde{f}| \leq 0.17 \times 10^{-10} \mathrm{GeV}^{-2}$ for the charged Higgs mass

greater than $70 \mathrm{GeV}$. For larger values of $m_{H}$, tighter constraints are placed on $|\tilde{f}|$. Cutting the photon energy $E_{\gamma}$ to be larger than $2.1 \mathrm{GeV}$ to ensure that the contribution is indeed due to the penguin diagram contribution considered here, the central value of the branching ratio is reduced slightly to $2.97 \times 10^{-4}$. The allowed range, at fixed $\tilde{f}$, for the Higgs mass is restricted at the upper end by this reduction. For example, $|\tilde{f}|=0.17 \times 10^{-10} \mathrm{GeV}^{-2}$ is 
consistent with the reduced branching ratio for $70 \mathrm{GeV} \leq m_{H} \leq 110 \mathrm{GeV}$. There is a region in which the constraints on $\tilde{f}$ from $b \rightarrow s \gamma, \epsilon$ and the neutron EDM are consistent. But it is not possible to simultaneously satisfy the constraints from $b \rightarrow s \gamma$ and from $\epsilon^{\prime} / \epsilon$.

\section{DISCUSSION}

No constraint is yet placed on the model by the results for $\sin 2 \beta$ from ALEPH, OPAL, CDF, BaBar or Belle.

If the current quark mass $m_{d} \sim 10 \mathrm{MeV}$ is used, the resulting value of $d_{n}$ satisfies the experimental limit as long as $\tilde{f}<2.56 \times 10^{-10} \mathrm{GeV}^{-2}$. However, if constituent mass $m_{d} \sim 300 \mathrm{MeV}$ is used, the model may be in trouble. We know of no convincing argument for preferring one mass over the other, and therefore conservatively use the current quark mass to estimate limits.

The values of $\tilde{f}$ from $\epsilon^{\prime} / \epsilon, \epsilon$ and $d_{n}$ then have a region of consistency, as do the values constrained by $B(b \rightarrow s \gamma), \epsilon$ and $d_{n}$.

However there is a definite conflict between the limits on $\tilde{f}$ from $\epsilon^{\prime} / \epsilon$ and $B(b \rightarrow s \gamma)$. The latter requires $|\tilde{f}| \leq 0.17 \times 10^{-10} \mathrm{GeV}^{-2}$, and the former requires $0.35 \times 10^{-10} \mathrm{GeV}^{-2} \leq$ $\tilde{f} \leq 3.1 \times 10^{-10} \mathrm{GeV}^{-2}$. As we have been careful to make very conservative estimates of the

allowed range of $\tilde{f}$ (in the hope of finding that there was still a small region of parameter space in which the model is consistent with the data), the gap between these allowed regions for $\tilde{f}$ is unbridgeable. Thus we conclude that the Weinberg model is ruled out by the recent data for $\epsilon^{\prime} / \epsilon$ and for $B(b \rightarrow s \gamma)$.

One of the attractive features of the version of Weinberg model we discuss here is that $\mathrm{CP}$ violation is generated spontaneously, rather than being put in by hand. If we abandon this attractive feature, and explicit CP violation is introduced into the Higgs interaction, as in Eq (1), as well as into the $\mathrm{W}$ interaction by a phase in the KM matrix, this new model (which we will call the modified Weinberg model) is not ruled out. The constraint from $b \rightarrow s \gamma$ requires that the contribution to $\epsilon^{\prime} / \epsilon$ from the Higgs interaction is small, and the 
main contribution to this CP violating parameter is just the same as that of the SM. As has been pointed out in Ref. [1] there are large uncertainties in the SM calculations due to our poor understanding of the hadronic matrix elements. One can find allowed regions in parameter space in which the experimental value for $\epsilon^{\prime} / \epsilon$ is produced in the SM, and thus in the modified Weinberg model as well. On the other hand, since the charged Higgs exchange can also contribute appreciably to $\epsilon$ in this model and can partially cancel the KM contribution, as a result the KM phase can have a larger allowed range than that in SM and can easily accomodate the larger value of $\epsilon^{\prime} / \epsilon$. Also, in this model, the value of $d_{n}$ can still be as large as $3 \times 10^{-25}\left(m_{d} / 300 \mathrm{MeV}\right) \mathrm{em}$, very different from the tiny value of the SM [32]. And $\sin 2 \beta$ can take the large values characteristic of the SM.

\section{ACKNOWLEDGMENTS}

This work was supported in part by grants NSC 88-2112-M-002-041, and NSC 89-2112M-007-010 of the National Science Council of R.O.C., and in part by the Australian Reserach Council. DC wishes to thank the US Department of Energy for partial support while at

SLAC. BMcK thanks the Department of Physics of National Taiwan University for their hospitality. 


\section{REFERENCES}

[1] Y.-Y. Keum, U. Nieste and A. Sanda, Phys. Lett. B457, 157(1999); For recent review see for example, S. Bosch et al., Nucl. Phys. B565, 3(2000).

[2] Particle Data Group, Euro. Phys. J. C3, 103(1998).

[3] A. Alavi-Harati et al. (KTeV Collaboration), Phys. Rev. Lett. 83, 22(1999); A documentation of the NA48 result on $\epsilon^{\prime} / \epsilon$ can be found at: http://www.cern.ch/NA48.

[4] A.G. Cohen, D.B. Kaplan and A.E. Nelson, Ann. Rev. Nucl. Part. Sci. 43, 27(1993).

[5] T. D. Lee, Phys. Rev. D8, 1226(1973); Phys. Rep. 96, 143(1974).

[6] S. Weinberg, Phys. Rev. Lett. 37, 657(1976).

[7] H.-Y. Cheng, Int. J. Mod. Phys. A7, 1059(1992).

[8] G. C. Branco, Phys. Rev. Lett. 44, 504(1980).

[9] P. Krawczyk and S. Pokorski, Nucl. Phys. B364, 10(1991).

[10] Y. Grossmann and Y. Nir, Phys. Lett. B313, 126(1993).

[11] H. Aihara (Belle Collaboration); D. Hitlin (BaBar Collaboration), in Proceeding od the XXXth Int. Conf. On High Energy Phyiscs, Osaka, Japan, July 27 - August 2, 2000.

[12] R. Forty, Aleph collaboration, Aleph 99-099/CONF 99-054.

[13] A. Sanda, Phys. Rev. D23, 2647(1981); N. Deshpande, ibid 23, 2654(1981);

[14] J. Donoghue, J. Hagelin and B. Holstein, Phys. Rev. D25, 195(1982).

[15] Y. Dupont and T.N. Pham, Phys. Rev. D28, 2169(1983); D. Chang, Phys. Rev. D25, 1318(1982); J. Hagelin, Phys. Lett. B117, 441(1982).

[16] J. Donoghue and B. Holstein, Phys. Rev. D32, 1152(1985); H.-Y. Cheng, Phys. Rev. D34, 1397(1986). 
[17] I.I. Bigi and A. Sanda, Phys. Rev. Lett. 58, 1604(1987); I. B. Khriplovich, Phys. Lett. B382, 145(1996).

[18] In fact in some cases the uncertainty is so large that it seems more appropriate to refer to estimates rather than calculations of the observables.

[19] S. Glenn et al., CLEO Collaboration, CLEO CONF 98-17; M. Nakao, Belle Collaboration, in Proceedings of the XXXth Int. Conf. On High Energy Physics, Osaka, Japan, July 27 - August 2, 2000.

[20] C. Albright, J. Smith and S.H.H. Tye, Phys. Rev. D21, 711(1980); K. Shizuya and S.-H. Tye, Phys. Rev. D23, 1613(1981); D. Chang, Phys. Rev. D25, 1318(1982).

[21] M. Chiuchini et al., Phys. Lett. B316, 127(1993).

[22] N. Deshpande, X.-G. He and S. Pakvasa, Phys. Lett. B326, 307(1994).

[23] S. Bertolini, M. Fabbrichesi and E. Gabrielli, Phys. Lett. B327,136(1994).

[24] J. Donoghue et al., Phys. Lett. B179, 361(1986); A. Buras and J. Gerard, Phys. Lett. B192, 156(1987).

[25] X.-G. He and G. Valencia, Phys. Rev. D61, 117501(2000).

[26] S. Weinberg, Phys. Rev. Lett. 63, 2333(1989).

[27] T. DeGrand et al., Phys. Rev. D12, 2060(1975); J. Donoghue, E. Golowich and B. Holstein, Phys. Rev. D15, 1341(1977).

[28] T. Feldmann, P. Kroll and B. Stech, Phys. Rev. D48, 114006(1998); E. Venugopal and B. Holstein, Phys. Rev. D57, 4397(1998).

[29] J. Donoghue, B. Holstein and Y.C. Lin, Nucl. Phys. B277, 651(1986).

[30] H.-Y. Cheng, Phys. Lett. B245, 122(1990).

[31] N. Deshpande and E. Ma, Phys. Rev. D16, 1583(1977). A. Anselm et al., Phys. Lett. 
B152, 116(1985).

[32] X.-G. He, B. H. J. McKellar and S. Pakvasa, Int. J. Mod. Phys. A4, 5011(1989).

[33] S. Weinberg, Phys. Rev. Lett. 63, 2333(1989); D. Dicus, Phys. Rev. D41, 999(1990).

[34] S. Barr and A. Zee, Phys. rev. Lett. 65, 21(1990); D. Chang, W.Y. Keung and T.C. Yuan, Phys. Lett. B251, 608(1990); J. Gunion and D. Wuler, Phys. Lett. B248, 170(1990); X.-G. He, B. H. J. McKellar and S. Pakvasa, Phys. Lett. B254, 231(1991); X.-G. He and B. H. J. McKellar, Phys. Rev. D46. 2131(1992); D. Bowser-Chao, D. Chang and W.Y. Keung, Phys. Rev. Lett. 79, 1988(1997).

[35] M. Hecht and B. H. J. McKellar, Phys.Rev. C60, 065202 (1999).

[36] M. Ciuchini, E. Gabrielli and G. Giudice, Phys. Lett. B388, 353(1996); Erratum-ibid B393, 489(1997). 